\title{
Pulse Wave Velocity as a Measure of Arterial Stiffness in Patients with Abdominal Aortic Aneurysm: A Systematic Review and Meta-Analysis
}

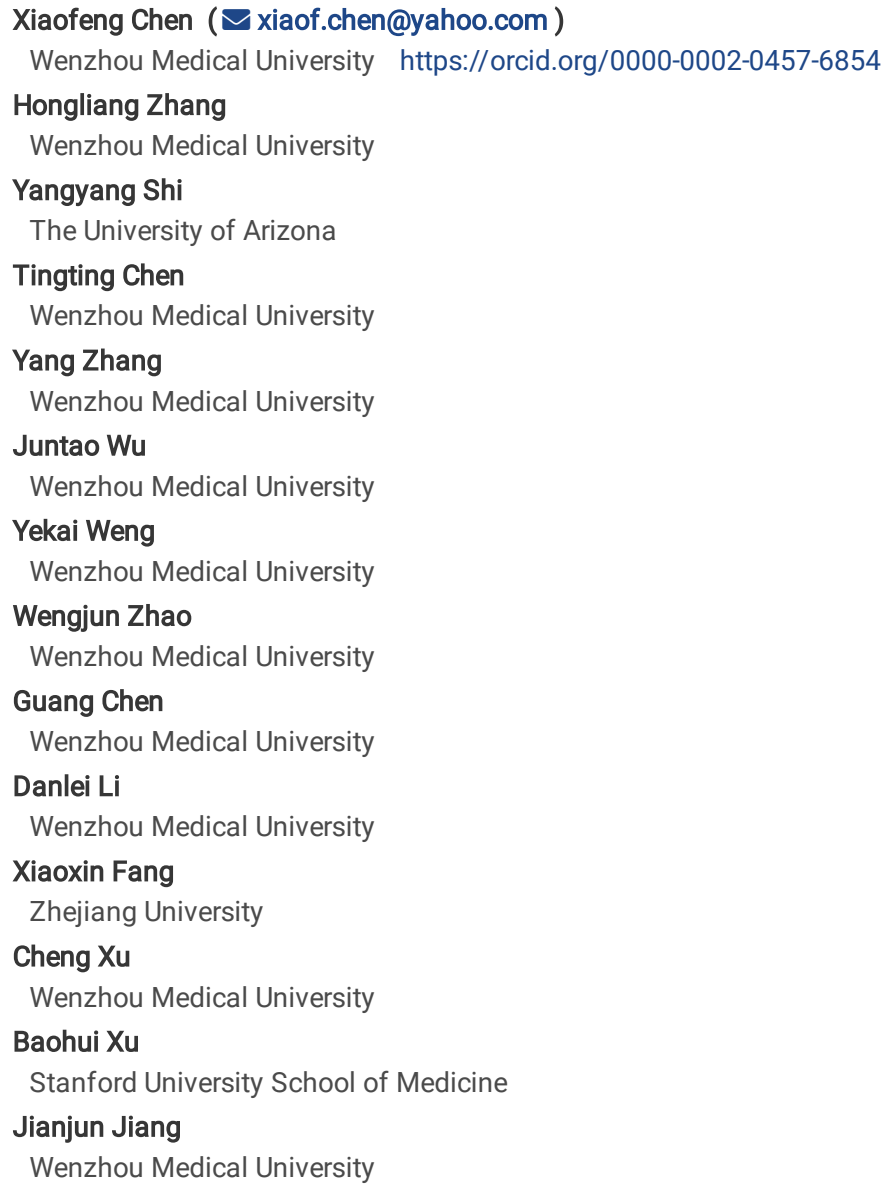

Research

Keywords: Pulse wave velocity, Abdominal aortic aneurysm, Meta-analysis

Posted Date: June 22nd, 2021

DOI: https://doi.org/10.21203/rs.3.rs-615922/v1

License: (a) (i) This work is licensed under a Creative Commons Attribution 4.0 International License. Read Full License 


\section{Abstract \\ Background and objective:}

The relationship between pulse wave velocity (PWV) levels and abdominal aortic aneurysm (AAA) remains controversial. A meta-analysis was performed to establish whether vascular pulse wave velocity (PWV) as a measure of arterial stiffness is different in patients with abdominal aortic aneurysms and controls.

\section{Methods}

Pubmed, Embase, Cochrane and China National Knowledge Infrastructure (CNKI) were used for the meta-analysis with articles up to January 1, 2021. To compare PWV levels between AAA patients and healthy controls, pooled weighted mean difference (WMD) and its $95 \%$ confidence interval (Cl) were calculated. Subgroup analysis and funnel plots are used to assess the quality of the combined results to ensure a normal distribution of data with minimal bias. Study quality for eligible studies was assessed using the Agency For Health Care Research and Quality (AHRQ) inventory tool.

\section{Results}

Nine cross-sectional studies, which included 439 abdominal aortic aneurysm cases and 382 healthy subjects, met inclusion criteria and were eligible for metaanalysis. We found that PWV levels were significantly higher [WMD $(95 \% \mathrm{Cl}): 2.36(2.02,2.70)]$ in AAA patients than healthy controls. After subgroup analysis, it was found that age, sex, smoking and hypertension had significant effects on the PWV levels. The normal distribution of the Funnel plot analysis suggests a low risk for publication bias.

\section{Conclusion}

PWV levels were elevated in patients with AAA compared to healthy controls, with the effect on PWV altered by age, sex, smoking and hypertension. Our study suggests that abdominal aortic aneurysm is related to increased arterial stiffness.

\section{Introduction}

Abdominal aortic aneurysm(AAA) is defined as the localized dilatation of the aorta and is likely to rupture unexpectedly leading to serious morbidity and mortality $[1,2]$. It is estimated that the incidence of AAA is about $4-8 \%$ in men and $1-1.3 \%$ in women and globally accounts for 168,200 deaths annually $[3,4$, 5]. The primary mechanism by which AAA develop consists of chronic inflammation, vascular smooth muscle cell (VSMC) apoptosis, extracellular matrix (ECM) degradation, and thrombosis. [6, 7] Specifically, these factors are what contributes to atherosclerotic plaque formation, and hence atherosclerosis plays a significant role in forming AAA and plays a role in the aneurysmal wall formation.[8]

Atherosclerosis of arteries contributes to the loss of arterial elasticity, elevated arterial stiffness, and therefore increased PWV since pulse velocity travels faster in stiffed arteries. $[9,10]$ Arterial stiffness (AS), represented by the arterial wall rigidity, is one of the most availably detectable manifestations of adverse structural and functional alterations within the arterial vessel wall.[11] Pulse wave velocity(PWV) is the velocity of a pulse wave moving through an arterial segment and is one of the non-invasive methods of assessing AS. $[12,13]$ The current literature on the differences between the PWV of AAA and controls is mixed. In some studies, PWV levels were significantly lower in AAA patients than those in healthy controls. [14] Contrarily, other studies have demonstrated that PWV levels in the abdominal aorta were significantly higher in AAA patients than those in non-AAA controls. [15, 16] Additional studies have failed to confirm either association of PWV levels with AAA.[17]

Based on the mixed literature in regards to the available evidence for pulse wave velocity and AAA incidence, we hypothesized that there may be a correlation between PWV levels and AAA. Therefore, we carried out a meta-analysis using published studies on PWV levels in AAA patients.

\section{Material And Method}

\section{Database and Search Strategies}

Our meta-analysis strictly followed the Preferred Reporting Items for Systematic Review and Meta-analysis (PRISMA) guidelines[18].

We systematically searched PubMed, Embase, Cochrane Central Register of Controlled Trials, and the Chinese National Knowledge Infrastructure (CNKI database). The Medical Subject Headings (Mesh) and relevant keywords were used for literature research. The electronic literature search was complemented by a manual search of related articles. The reference lists of collected items were also manually reviewed for additional correlated articles.

PubMed search strategy was conducted using the following search field descriptions and tags; Title/Abstract [tiab] and MeSH Terms [MH]

\#1."pulse wave velocity [mh]" OR "PWV [tiab]" OR "aPWV [tiab]" OR "cPWV [tiab]" OR "fPWV [tiab]" OR "cfPWV [tiab]" OR "arterial distensibility [tiab]" OR "vascular distensibility [tiab]" OR "aortic distensibility [tiab]" OR "arterial stiffness [tiab]" OR "arterial stiffening [tiab]" OR "vascular stiffness [tiab]" OR "vascular stiffening [tiab]" OR "aortic stiffness [tiab]" OR "aortic stiffening [tiab]" OR "arterial compliance [tiab]" OR "vascular compliance [tiab]" OR "aortic compliance [tiab]" 
\#2. "abdominal aortic aneurysm [mh]" OR "Aneurysms, Abdominal aortic [tiab]" OR "Aortic Aneurysms, Abdominal [tiab]" OR "Abdominal Aortic Aneurysm [tiab]" OR "Aneurysm, Abdominal Aortic [tiab]"

\section{Inclusion and exclusion criteria of enrolled studies}

Our inclusion criteria were as follows: the study had to be observational (i.e., a case-control, cross-sectional or cohort design); the subjects enrolled had to be diagnosed by the physician as AAA; there had to be comparison of vascular PWV between patients with AAA and controls without AAA; and PWV data had to be available for all patients. Exclusion criteria included: study had undefined control groups, duplicate or overlapped populations with a previous study, or PWV values were only reported in a single group and not both AAA and control.

\section{Data extraction}

Data were independently extracted from individual studies by two reviewers. Each study's information was obtained using standardized forms by two independent observers blinded to the authors' names and journal titles. Discrepancies between the outputs were resolved through discussion and involvement of a third co-author. The following information was extracted from each study (Table 1): (I) trial's name/publication year; (II)numbers of subjects enrolled; (III)country; (V)age; (VI)male; (VII)BMI; (VIII)outcome index; (IX)PWV levels; (X)device; (XI) AHRQ scores; (XII)Smoking; (XIII)Hypertension; (XV)diabetes.

\section{Assessment of the risk for publication bias}

This was conducted by two researchers independently. Because this meta-analysis included cross-sectional studies, study quality was assessed using the Agency For Health Care Research and Quality (AHRQ) inventory tool[19]. Eleven questions were answered. If the answer was "No" or "Unclear," the rating of the item was "1"; if the answer was "Yes," the score of the item was "1". The quality for each study was scored as low (0-3), medium (4-7) or high (8-11). Studies graded as low quality (0-3) were excluded from the meta-analysis.

\section{Statistical analysis}

The meta-analysis was conducted using a synthesis dataset according to published eligible studies. Weighted Mean Difference (WMD) with corresponding $95 \%$ confidence interval $(\mathrm{Cl})$ was calculated to evaluate PWV and AAA correlations. If the $\mathrm{I}^{2}$ value was $>50 \%$ with a $p<0.1$ for the test of heterogeneity, the results were considered heterogeneous.

Forest plots were used to display the results graphically. Further subgroup analysis based on anatomic location of PWV (carotid-femoral PWV and aortic PWV) was performed since some studies have argued that PWV in different locations of stiffened arteries varied unexpectedly. Additional subgroup analyses included age, sex, smoking, diabetes and hypertension. Subgroup analysis was also used to identify the factors contributing to the heterogeneity of the pooled data.

All reported $\mathrm{P}$ values were two-sided tests and considered statistically significant if $\mathrm{P}<0.05$. Publication bias was assessed using funnel plots. All statistical analyses were performed by Stata 12.0 (Stata Corporation, College Station, TX, USA).

\section{Results}

\section{Literature search}

The literature and eligible studies are detailed in Figure 1. A preliminary electronic database search identified 244 potential records. After reviewing abstracts or full articles, 103 duplicate articles, 126 unrelated articles, 2 review articles, and 3 articles with unextractable data were excluded. In the end, nine studies qualified for the meta-analysis examining the association of PWV with AAA.

\section{Study characteristics}

The characteristics of included studies are presented in Table 1.6 of which reported carotid-femoral PWV (cf-PWV) and 3 of which reported infrarenal arterial aortic PWV. Four studies reported data on both males and females, while 3 studies only reported data on males.

\section{PWV measurement methods}

Methods for PWV measurements varied among studies. 2 studies used a Complier SP, 2 studies used a Sphygmocor device, 2 studies used a Sonix RP system, and 3 studies used Electrocardiographically Gated 64-Detector Row CT.

\section{Association of PWV levels with AAA}

All 9 eligible studies reported on the relationship of PWV levels with AAA. As quantified using $\mathrm{I}^{2}$ and P-value, the heterogeneity across included studies was statistically significant $\left(P<0.00001, I^{2}=98.9 \%\right)$. Studies were weighted based on the number of patients in each study and other factors. Meta-analysis showed that patient's PWV values were higher in patients with AAA than those in control subjects (WMD $=2.36 \mathrm{~m} / \mathrm{s}, 95 \% \mathrm{Cl} 2.02 \varangle 2.70 \rrbracket \mathrm{P} \llbracket 0.001)(\mathrm{Figure} 2 \mathrm{~A})$. To investigate potential publication bias, the funnel plot was created for studies included in a meta-analysis of PWV (Figure 2B), with a vertical axis for study size (standard error) and horizontal axis for the standardized difference in means which averaged overall at around 2.36. Lack of significant asymmetry in the funnel plot both on the $x$ and $Y$ axis in Figure 2 B suggests the low likelihood of publication bias.

\section{Subgroup analysis}


Subgroup meta-analysis results are summarized in Table 2. High levels of pulse wave velocity were associated with AAA as defined by cf-PWV $(W M D=0.63 \mathrm{~m} / \mathrm{s}, 95 \% \mathrm{Cl}$ 0.26-1.00, $\mathrm{P} \otimes 0.00001)$ and infrarenal $\mathrm{PWV}(\mathrm{WMD}=12.65 \mathrm{~m} / \mathrm{s}, 95 \% \mathrm{Cl} 11.75-13.54, \mathrm{P}=0.004)$ (Figure 3B).

Subgroup analysis was also performed if it was possible to match risk factors such as age (9/9 studies), sex (7/9 studies), smoking(6/9 studies), diabetes(5/9 studies) and hypertension(5/9 studies) (Table 2$)$.

The PWV levels were significantly lower in AAA patients than those in the control group if there was significant inter-group difference in age between AAA and control (WMD:-0.48; 95\% Cl:-0.94,-0.01, $\mathrm{I}^{2}=77 \%, \mathrm{P}=0.005$ ), however, $\mathrm{PWV}$ levels were significantly higher in AAA patients than those in the control group if there was not significant inter-group difference in age between AAA and control (WMD:5.59; 95\%Cl:5.09,6.09, $\left.\mathrm{I}^{2=} 99 \%, \mathrm{P}<0.000\right)(\mathrm{Figure} 3 \mathrm{~B}$ ).

The PWV levels were significantly lower in AAA patients than those in the control group if there was significant inter-group difference in hypertension between AAA and control(WMD:-0.52;95\% Cl:-0.99,-0.06, $\left.\mathrm{I}^{2}=88.8 \%, \mathrm{P}=0.003\right)$, however, $\mathrm{PWV}$ levels were significantly higher in AAA patients than those in the control group if there was not significant inter-group difference in hypertension between AAA and control (WMD:2.05; 95\%Cl:1.23,2.87, $\left.I^{2}=96.7 \%, P<0.000\right)($ Figure $4 A)$.

The PWV levels were significantly lower in AAA patients than those in the control group if there was significant inter-group difference in diabetes between AAA and control(WMD:-2.10; 95\%Cl:-3.23,-0.97), however, PWV levels were significantly higher in AAA patients than those in the control group if there was not

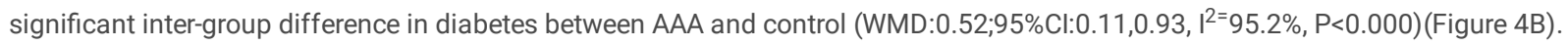

The PWV levels were not significantly different in AAA patients than those in the control group if there was significant inter-group difference in sex between AAA and control(WMD:-0.20; 95\% Cl:-0.71,0.31), however, PWV levels were significantly higher in AAA patients than those in the control group if there was not significant inter-group difference in sex between AAA and control (WMD:4.35;95\%Cl:3.89, 4.80, $1^{2=99.1 \%, P<0.000)(F i g u r e ~ 5 A) . ~}$

The PWV levels were significantly higher in AAA patients than those in the control group if there was significant inter-group difference in smoking between AAA and control(WMD:1.12; $95 \% \mathrm{Cl}: 0.44,1.81)$, PWV levels were also significantly higher in AAA patients than those in the control group if there was not significant inter-group difference in smoking between AAA and control (WMD: $0.43 ; 95 \%$ Cl: $-0.00,0.87,1^{2=95.2 \%, P<0.000)(F i g u r e ~ 5 B) . ~}$

\section{Discussion}

This meta-analysis investigated whether vascular PWV levels as a measure of arterial stiffness is different in patients with abdominal aortic aneurysms compared to controls. A total of 9 cross-sectional studies met inclusion criteria for our analysis. Our results demonstrated that PWV levels in patients with AAA were significantly higher than those in controls, indicating the relationship between the abdominal aortic aneurysm and increased arterial stiffness.

There are several mechanisms by which atherosclerosis of arteries contributes to abdominal aortic aneurysms. Both conditions are multifactorial diseases with known risk factors that contribute toward disease development. Carotid atherosclerosis is positively correlated to the growth of aortic diameter and aneurysmal formation.[20] Pulse wave velocity (PWV) is assumed to be the velocity of a pulse wave moving through an arterial segment. This meta-analysis shows that the PWV levels of AAA patients are higher than those of the control group, indicating that abdominal aortic aneurysm is related to increased arterial stiffness. Vascular PWV as a measure of arterial stiffness is changed in patients with AAA. Aortic stiffness, the manifestation of pulse wave velocity, is associated with the change in the structural protein of the medial extracellular matrix, such as elastin and collagen.[21, 22] Elevated blood pressure and repetitive cyclic stress leads to the wearing out of the elastic components of the aortic walls[23], which leads to the stiffening and dilation of the aortic wall. Furthermore, the inflammatory process within the arterial wall, accompanied by invasion of monocytes and macrophages and increased levels of IL-6, can accelerate the formation and development of AAA.[24] A series of published literature support the association of inflammatory markers with arterial stiffness in patients with cardiovascular diseases.[25] Finally, calcium deposition in the AAA wall results in increased arterial stiffness in AAA.[26]

Meta-analysis showed that patients' PWV values were higher in patients with AAA than those in control subjects $(\mathrm{WMD}=2.36 \mathrm{~m} / \mathrm{s}, 95 \% \mathrm{Cl} 2.02 \varangle 2.70, \mathrm{P} \otimes 0.001)$. The subgroup meta-analyses of smoking and detection location supported the result that the PWV levels were significantly higher in AAA patients. However, there were unexpected findings in the subgroup meta-analyses of age, diabetes, hypertensive and sex. These may possibly due to the heterogeneity among the studies. Subgroup analysis found that several factors influenced the effect sizes in PWV between AAA and control subjects (Table 2). Age, smoking and hypertension promote atherosclerosis, vascular wall medial degeneration and ultimately alter PWV [27]. It has been shown that elevated blood pressure worsens arterial stiffness indirectly. [28, 29] Arterial stiffness (change of PWV) also triggers loop feedback on systolic blood pressure (afterload) by accelerating wave reflection. [30.31.32] For example, the rate of increase in cf-PWV with pre-hypertension (systolic BP 120-139 mmHg) or hypertension(systolic BP $\geq 140 \mathrm{mmHg}$ ) is steeper compared with men with normal systolic BP (systolic BP $<120 \mathrm{mmHg}$ ). [33] In contrast, advancing age is predominantly associated with reduced arterial distensibility and thus aortic compliance. Cf-PWV increased monotonically before age $50-60$ years and then exponentially thereafter. [34] Additionally, smoking was also an independent predictor of adult PWV and biomarkers of endothelial function[35], all critically affecting PWV as well as AAA.

There are several limitations of our meta-analysis. First, only 9 cohort studies of PWV about abdominal aortic aneurysms were enrolled for the final analysis due to inclusion criterias. Because some included studies were cross-sectional, we cannot rule out the possibility of selection bias. Second, the different PWV location detection methods renders our study a certain degree of lack of reliability. Current arterial stiffness methods include the local measurement of arterial stiffness using ultrasonography and magnetic resonance imaging and regional measurement by tonometer and Doppler probes.[36] Due to the limited number of studies, we included all the studies with different PWV measurement methods. Therefore, variation inevitably exists and cannot be avoided, and we should also keep in mind the variability of measuring each individual patient. Third, the presence of other risk factors and publication bias suggests that the analysis results may not be entirely accurate. These limitations may potentially influence the reliability and accuracy of our results. 
In conclusion, our meta-analysis showed that PWV levels in AAA patients were significantly higher than those in control subjects. PWV value as a measure of arterial stiffness is changed in patients with abdominal aortic aneurysm and may be used to monitor disease progression. Our analysis warrants further investigation into the the association of PWV levels with AAA disease in a more well-designed prospective cohort study.

\section{Declarations}

\section{Ethical Approval and Consent to participate}

Not applicable

\section{Consent for publication}

Not applicable

\section{Availability of data and materials}

Not applicable.

\section{Competing interests}

The authors declare that they have no competing interests

\section{Funding}

This study was supported by the grant from National Natural Science Foundation, China (N. 81770475).

\section{Authors' contributions}

HLZ, YYS, TTC contribute to the conception and design of the study, HLZ, YYS,TTC, YZ, JTW, YKW, WJZ, GC, DLL, XXF, CX, BHX, JJJ and XFC contribute to analysis and interpretation of data, HLZ and XFC contribute to drafting the article, All of authors revise it critically for important intellectual content, and final approval of the version to be published.

\section{Acknowledgements}

Not applicable

\section{Authors' information}

Not applicable

\section{References}

1. Aggarwal S, Qamar A, Sharma V, Sharma A. Abdominal aortic aneurysm: A comprehensive review. Exp Clin Cardiol. 2011;16(1):11-15.

2. Johnston KW, Rutherford RB, Tilson MD, Shah DM, Hollier L, Stanley JC. Suggested standards for reporting on arterial aneurysms. Subcommittee on Reporting Standards for Arterial Aneurysms, Ad Hoc Committee on Reporting Standards, Society for Vascular Surgery and North American Chapter, International Society for Cardiovascular Surgery. Journal of vascular surgery. 1991;13(3):452-458.

3. Bird AN, Davis AM. Screening for abdominal aortic aneurysm. Jama. 2015;313(11):1156-1157.

4. Global, regional, and national life expectancy, all-cause mortality, and cause-specific mortality for 249 causes of death, 1980-2015: a systematic analysis for the Global Burden of Disease Study 2015. Lancet (London, England). 2016;388(10053):1459-1544.

5. Global, regional, and national disability-adjusted life-years (DALYs) for 315 diseases and injuries and healthy life expectancy (HALE), 1990-2015: a systematic analysis for the Global Burden of Disease Study 2015. Lancet (London, England). 2016;388(10053):1603-1658.

6. Wassef M, Baxter BT, Chisholm RL, Dalman RL, Fillinger MF, Heinecke J, et al. Pathogenesis of abdominal aortic aneurysms: A multidisciplinary research program supported by the National Heart, Lung, and Blood Institute. J Vasc Surg 2001;34:730-8.

7. Pearce WH, Shively VP. Abdominal aortic aneurysm as a complex multifactorial disease: interactions of polymorphisms of inflammatory genes, features of autoimmunity, and current status of MMPs. Ann N Y Acad Sci 2006;1085:117-32

8. Abdominal aortic aneurysm-an independent disease to atherosclerosis? $₫ 2017$ Bradley J. Toghill $\rrbracket$, Athanasios Saratzis, Matthew J. Bown

9. Laurent S, Cockcroft J, Van Bortel L, et al. Expert consensus document on arterial stiffness: methodological issues and clinical applications. European heart journal. 2006;27(21):2588-2605

10. Achimastos AD, Efstathiou SP, Christoforatos T, Panagiotou TN, Stergiou GS, Mountokalakis TD.Arterial stiffness: determinants and relationship to the metabolic syndrome. Angiology. 2007;58(1):11-20.

11. Schwartz JE, Feig PU, Izzo JL Jr. Pulse wave velocities derived from cuff ambulatory pulse wave analysis. Hypertension 2019;74:111e116

12. Vlachopoulos C, Aznaouridis K, Stefanadis C. Prediction of cardiovascular events and all-cause mortality with arterial stiffness: a systematic review and meta-analysis. Journal of the American College of Cardiology. 2010;55(13):1318-1327.

13. Yannoutsos A, Bahous SA, Safar ME, Blacher J. Clinical relevance of aortic stiffness in end-stage renal disease and diabetes: implication for hypertension management. Journal of hypertension. 2018;36(6):1237-1246. 
14. Abbas A, Cecelja M, Hussain T, et al. Thoracic but not abdominal phase contrast magnetic resonance-derived aortic pulse wave velocity is elevated in patients with abdominal aortic aneurysm. Journal of hypertension. 2015;33(5):1032-1038.

15. Li L, Zha Y, Lu X, et al. A Preliminary Study of Assessing Aortic Elasticity in Infrarenal Abdominal Aortic Aneurysm With Electrocardiographically Gated 64Detector Row CT. 2016;35(06):882-886.

16. Durmus I, Kazaz Z, Altun G, Cansu A. Augmentation index and aortic pulse wave velocity in patients with abdominal aortic aneurysms. International journal of clinical and experimental medicine. 2014;7(2):421-425.

17. Bailey MA, Davies JM, Griffin KJ, et al. Carotid-femoral pulse wave velocity is negatively correlated with aortic diameter. Hypertension research : official journal of the Japanese Society of Hypertension. 2014;37(10):926-932.

18. Moher D, Liberati A, Tetzlaff J, Altman DG. Preferred reporting items for systematic reviews and meta-analyses: the PRISMA statement. International journal of surgery (London, England). 2010;8(5):336-341

19. Zeng X, Zhang Y, Kwong JS, et al. The methodological quality assessment tools for preclinical and clinical studies, systematic review and meta-analysis, and clinical practice guideline: a systematic review. Journal of evidence-based medicine. 2015;8(1):2-10.

20. S H Johnsen 1, S H Forsdahl, S Solberg, K Singh, B K Jacobsen. Carotid atherosclerosis and relation to growth of infrarenal aortic diameter and follow-up diameter: the Troms $\emptyset$ Study. 2013;45(2):135-40.

21. Cecelja M, Chowienczyk P. Role of arterial stiffness in cardiovascular disease. JRSM Cardiovasc Dis 2012; 1:1-26.

22. Lakatta EG, Mitchell JH, Pomerance A, Rowe GG. Human aging: changes in structure and function. J Am Coll Cardiol 1987; 10 (2 Suppl A):42A-47A

23. O’Rourke MF, Mancia G. Arterial stiffness. J Hypertens 1999; 17:1-4.

24. Dawson J,Cockerill GW, Choke E, Belli AM, Loftus I,Thompson MM. Aortic aneurysms secrete interleukin-6 into the circulation. J Vasc Surg 2007;45:350-6.

25. Ikonomidis I, Stamatelopoulos K, Lekakis J, Vamvakou GD, Kremastinos DT. Inflammatory and non-invasive vascular markers: the multimarker approach for risk stratification in coronary artery disease. Atherosclerosis 2008;199:3-11.

26. Rentschler ME, Baxter BT. Screening aortic drug treatments through arterial compliance measurements. Curr Vasc Pharmacol 2008;6:250-7.

27. Avolio AP, Chen SG, Wang RP, Zhang CL, Li MF, O'Rourke MF. Effects of aging on changing arterial compliance and left ventricular load in a northern Chinese urban community. Circulation. 1983;68(1):50-58.

28. O'Rourke MF, Mancia G. Arterial stiffness. Journal of hypertension. 1999;17(1):1-4.

29. Avolio AP, Chen SG, Wang RP, Zhang CL, Li MF, O'Rourke MF. Effects of aging on changing arterial compliance and left ventricular load in a northern Chinese urban community. Circulation. 1983;68(1):50-58.

30. Kaess BM, Rong J, Larson MG, et al. Aortic stiffness, blood pressure progression, and incident hypertension. Jama. 2012;308(9):875-881.

31. Benetos A, Adamopoulos C, Bureau JM, et al. Determinants of accelerated progression of arterial stiffness in normotensive subjects and in treated hypertensive subjects over a 6-year period. Circulation. 2002;105(10):1202-1207.

32. Takase H, Dohi Y, Toriyama T, et al. Brachial-ankle pulse wave velocity predicts increase in blood pressure and onset of hypertension. American journal of hypertension. 2011;24(6):667-673.

33. AlGhatrif M, Strait JB, Morrell CH, et al. Longitudinal trajectories of arterial stiffness and the role of blood pressure: the Baltimore Longitudinal Study of Aging. Hypertension (Dallas, Tex : 1979). 2013;62(5):934-941.

34. Mitchell GF, Parise H, Benjamin EJ, et al. Changes in arterial stiffness and wave reflection with advancing age in healthy men and women: the Framingham Heart Study. Hypertension (Dallas, Tex : 1979). 2004;43(6):1239-1245.

35. van de Laar RJ, Stehouwer CD, Boreham CA, et al. Continuing smoking between adolescence and young adulthood is associated with higher arterial stiffness in young adults: the Northern Ireland Young Hearts Project. Journal of hypertension. 2011;29(11):2201-2209.

36. Stephane Laurent, John Cockcroft, Luc Van Bortel, Pierre Boutouyrie, Cristina Giannattasio, Daniel Hayoz, Bruno Pannier, Charalambos Vlachopoulos, lan Wilkinson, Harry Struijker-Boudier, European Network for Non-invasive Investigation of Large Arteries Expert consensus document on arterial stiffness: methodological issues and clinical applications. Eur Heart J. 2006;27(21):2588-605.

\section{Tables}

Table 1: Baseline characteristics 


\begin{tabular}{|c|c|c|c|c|c|c|c|c|c|}
\hline Author/Year & $\mathrm{n}$ & Country & Age(year) & Male & $\mathrm{BMI}\left(\mathrm{kg} / \mathrm{m}^{2}\right)$ & Case/control & $\begin{array}{l}\text { Outcome } \\
\text { index }\end{array}$ & $\mathrm{PWV}(\mathrm{m} / \mathrm{s})$ & device \\
\hline Abbas, A.et & 88 & UK & AAA:73.1 \pm 5.7 & $46(100 \%)$ & $27.1 \pm 4.2$ & $46 / 42$ & cf-PWV & $10.8 \pm 2.2$ & Sphygmocor \\
\hline $\mathrm{Al} 2015^{[17]}$ & & & Control: $66.3 \pm 1$ & $42(100 \%)$ & $25.9 \pm 3.2$ & & & $12.9 \pm 3.1$ & device \\
\hline \multirow[t]{2}{*}{ Durmus, I.2014 ${ }^{[15]}$} & 38 & Turkey & AAA: $69 \pm 4$ & $11(61 \%)$ & $26.7 \pm 4.4$ & $18 / 20$ & cf-PWV & $14.8 \pm 4.9$ & Sphygmocor \\
\hline & & & Control: $66 \pm 6$ & $13(65 \%)$ & $29.5 \pm 4.4$ & & & $10 \pm 1.7$ & device \\
\hline \multirow[t]{2}{*}{ Bailey,M.A.2014 ${ }^{[16]}$} & 309 & UK & AAA: $73 \pm 7.5$ & $120(74.5 \%)$ & $28 \pm 4.6$ & $148 / 161$ & cf-PWV & $9.55 \pm 2.3$ & Vicoder \\
\hline & & & Control:69.7ะ7.8 & $128(86.4 \%)$ & $28 \pm 4.5$ & & & $9.75 \pm 2.3$ & device \\
\hline \multirow[t]{2}{*}{ Li, R. X.2013 ${ }^{[24]}$} & 20 & USA & NA & NA & NA & $5 / 15$ & Aortic & $10.54 \pm 6.52$ & A Sonix RP system \\
\hline & & & & & & & & $6.03 \pm 1.68$ & \\
\hline \multirow[t]{2}{*}{ Lee, C. W.2013 } & 102 & TaiWan & AAA:75.2 \pm 11.6 & $47(92.2 \%)$ & $23.8 \pm 4.2$ & $51 / 51$ & cf-PWV & $12.1 \pm 2.7$ & Colin VP-200 \\
\hline & & & Control:74.3 \pm 12.5 & $46(90.2 \%)$ & $24.9 \pm 3.4$ & & & $13.6 \pm 3.5$ & \\
\hline \multirow{2}{*}{$\begin{array}{l}\text { Kadoglou, N. P. } \\
\text { E.2012 }\end{array}$} & 149 & Greece & AAA: $72 \pm 4$ & $108(100 \%)$ & $28.98 \pm 4.23$ & $108 / 41$ & cf-PWV & $12.99 \pm 3.75$ & Complior SP, \\
\hline & & & Control:69 68 & $41(100 \%)$ & $29.36 \pm 5.79$ & & & $10.03 \pm 1.57$ & Alam Medical,Franct \\
\hline \multirow{2}{*}{$\begin{array}{l}\text { Kadoglou, N. P. } \\
\varangle 2 \otimes E .2012^{[22]}\end{array}$} & 79 & Greece & AAA: $71 \pm 4$ & $48(100 \%)$ & $28.6 \pm 4.2$ & $48 / 31$ & cf-PWV & $13.11 \pm 3.57$ & Complior SP, \\
\hline & & & Control: $69 \pm 4$ & $31(100 \%)$ & $27.9 \pm 3.5$ & & & $7.97 \pm 2.17$ & Alam Medical,Franct \\
\hline \multirow[t]{2}{*}{ Li, R. X.2011 ${ }^{[23]}$} & 10 & USA & NA & NA & NA & $2 / 8$ & Aortic & $10.54 \pm 6.52$ & A Sonix RP system \\
\hline & & & & & & & & $6.03 \pm 1.68$ & \\
\hline \multirow[t]{2}{*}{ Li L2016 ${ }^{[14]}$} & 26 & China & NA & $10(76.9 \%)$ & NA & $13 / 13$ & Aortic & $18.63 \pm 1.3$ & Electrocardiographi \\
\hline & & & & $10(76.9 \%)$ & & & & $5.7 \pm 1.06$ & \\
\hline
\end{tabular}

NA: not available

*PWV(Pulse wave velocity)

*AAA(abdominal aortic aneurysm)

${ }^{*}$ cf-PWV『carotid-femoral PWV,cfPWV)

*Aortic PWV(the infrarenal PWV)

Table 2: Subgroup analysis of PWV in AAA group and control group 


\begin{tabular}{|c|c|c|c|c|c|c|}
\hline \multirow[t]{2}{*}{ Subgroup } & \multirow[t]{2}{*}{$\mathrm{N}$} & \multirow[t]{2}{*}{ WMD $(95 \% \mathrm{Cl})$} & \multicolumn{2}{|c|}{ Test of SMD $=0$} & \multicolumn{2}{|c|}{ Heterogeneity } \\
\hline & & & Z & P For Z & $\mathrm{I}^{2}(\%)$ & $P$ for $I^{2}$ \\
\hline All studies & 9 & $2.23(2.02,2.70)$ & & & 98.9 & 0.000 \\
\hline \multicolumn{7}{|l|}{ Age between AAA and control } \\
\hline Significance difference & 4 & $-0.48(-0.94,-0.01)$ & 2.01 & 0.044 & 77.0 & 0.005 \\
\hline Not significance difference & 5 & $5.59(5.09,6.09)$ & 22.04 & 0.000 & 99.0 & 0.000 \\
\hline \multicolumn{7}{|c|}{ Diabete between AAA and control } \\
\hline Significance difference & 1 & $-2.10(-3.23,-0.97)$ & 3.63 & 0.000 & / & / \\
\hline Not significance difference & 4 & $0.52(0.11,0.93)$ & 2.49 & 0.013 & 95.2 & 0.000 \\
\hline \multicolumn{7}{|c|}{ Hypertensive between AAA and control } \\
\hline Significance difference & 2 & $-0.52(-0.99,-0.06)$ & 2.20 & 0.028 & 88.8 & 0.003 \\
\hline Not significance difference & 3 & $2.05(1.23,2.87)$ & 4.89 & 0.000 & 96.7 & 0.000 \\
\hline \multicolumn{7}{|l|}{ Sex between AAA and control } \\
\hline Significance difference & 1 & $-0.2(-0.71,0.31)$ & 0.76 & 0.445 & / & / \\
\hline Not significance difference & 6 & 4.35(3.89.4.80) & 18.73 & 0.000 & 99.1 & 0.000 \\
\hline \multicolumn{7}{|c|}{ Smoking between AAA and control } \\
\hline Significance difference & 2 & $1.12(0.44,1.81)$ & 3.23 & 0.001 & 98.0 & 0.000 \\
\hline Not significance difference & 4 & $0.43(-0.00,0.87)$ & 1.94 & 0.052 & 96.3 & 0.000 \\
\hline \multicolumn{7}{|l|}{ Detection location } \\
\hline cf-PWV & 6 & $0.63(0.26,1.00)$ & 3.37 & 0.001 & 96.2 & 0.000 \\
\hline Aortic PWV & 3 & $2.36(2.02,2.70)$ & 27.65 & 0.000 & 81.9 & 0.004 \\
\hline
\end{tabular}

*AAA(abdominal aortic aneurysm)

\section{Figures}




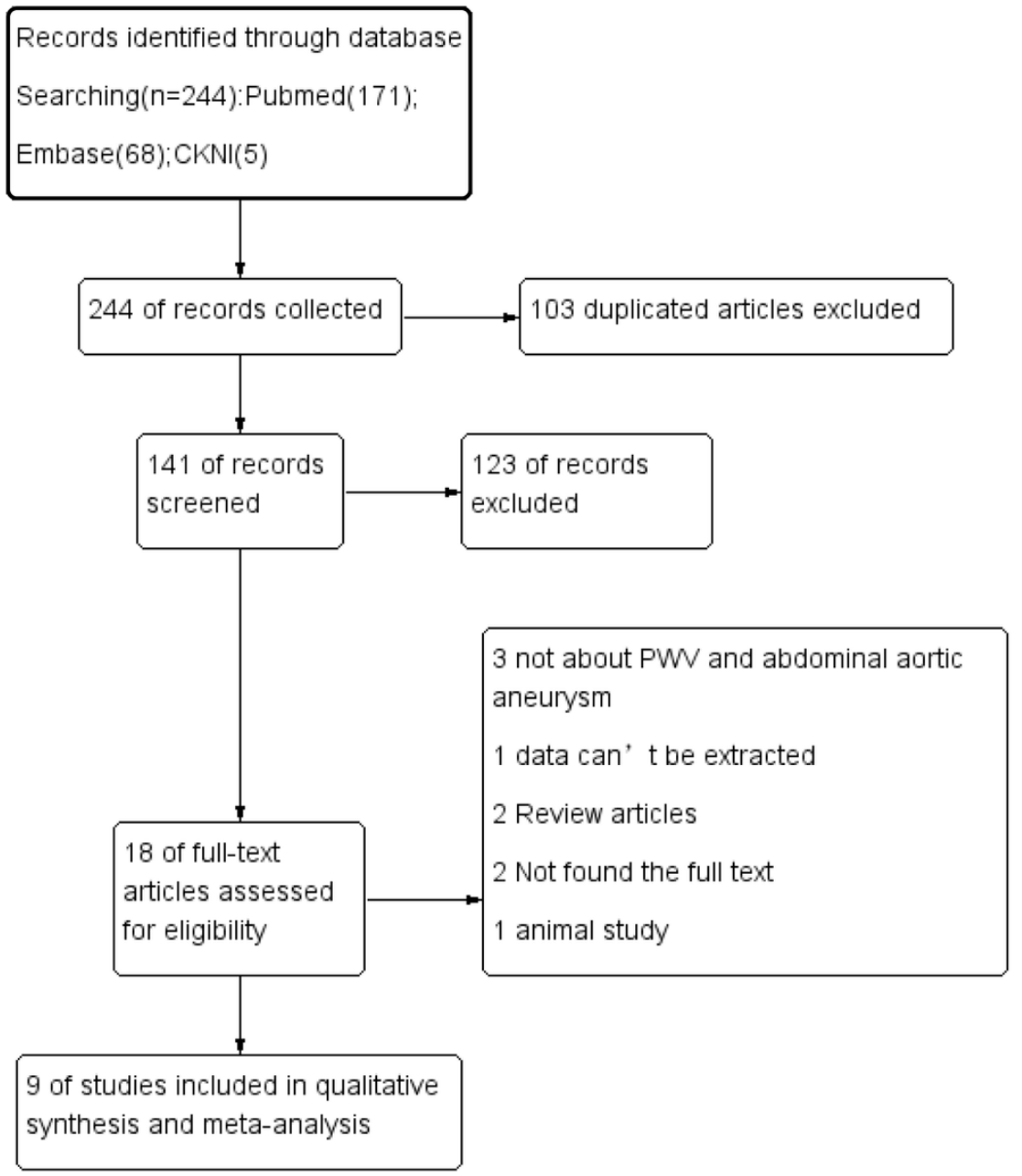

\section{Figure 1}

Flow diagram of the literature.

A

Study

ID

Abbas, Aet Al2015

Durmus, 1.2014

Bailey,M.A.2014

Li, R. X.2013

Lee, C. W.2013

Kadoglou, N. P. E.2012

Kadoglou, N.P. (2) E.2012

Li, R.X.2011

Li L2016

Overall (l-squared $=98.9 \%, p=0.000$ )

1

- 
A

Study

ID

a-PWN

Abbas, Aet A2015

Durmus, 1.2014

Bailey,MA.2014

Lee, C. W.2013

Kadoglou, N. P. E. 2012

Kadoglou, N. P. (2) E. 2012

Subtotal (1-squared $=96.2 \%, p=0.000$

Aortic PWV

Li, R. $\times 2013$

Li, R. $\times 2011$

LiL2016

Subtotal (1-squared $=81.9 \%, p=0.004$

Heterogenenity between yroupe: $p=0.000$

Overall (1-squared $=98.9 \%, p=0.000$ )

$\stackrel{1}{-13.8}$
WMD $(95 \% \mathrm{Cl}) \quad$ Weight

$-210(-323,-0.97) \quad 8.99$

$4.80(2.42 .7 .18) \quad 2.03$

$-0.20(-0.71,0.31) \quad 43.79$

$-1.50(-2.71,-0.29) \quad 7.84$

$296(2.10,3.82) \quad 1578$

$\begin{array}{ll}5.14(3.87,6.41) & 7.20 \\ 0.63(0.26,1.00) & 85.64\end{array}$

$4.51(-1.27,10.29) \quad 0.35$

$4.51(-4.60,13.62) \quad 0.14$

$12.93(12.02 .13 .84) \quad 13.88$

$12.65(11.75,13.54) \quad 14.36$

$2.36(2.02,2.70) \quad 100.00$
B

Studs

ID

WMD (95\% Cl) Weight

significant difference in age between $A A A$ and control

Abbas, Aet A22015

Bailey,MA.2014

L. R. $\times 2013$

L, R. $\times 2011$

Subtotal (1-squared $=77.0 \%, p=0.005$ )

not signiticant dimerence in age between AaA and control

Durmus, 12014

Lee, C. W.2013

Kadoglou, N. P. E 2012

Kadoglou, N. P. (2) E 2012

LiL2016

Subtotal (1-squared $=99.0 \%, p=0.000)$

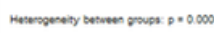

Overall (1-squared $=98.9 \%, p=0.000$

\begin{tabular}{l|lll} 
Overall (1-squared $=98.9 \%, p=0.000)$ & \multicolumn{2}{l}{$2.36(2.02,2.70)$} & 100.00 \\
\hline
\end{tabular}

\section{Figure 3}

Forest plots of meta-analysis data (A) subgroup analysis according to significant difference or not significant difference in age between AAA and control group; (B) subgroup analysis according to PWV measurement location (carotid-femoral PWV and aortic PWV).

A

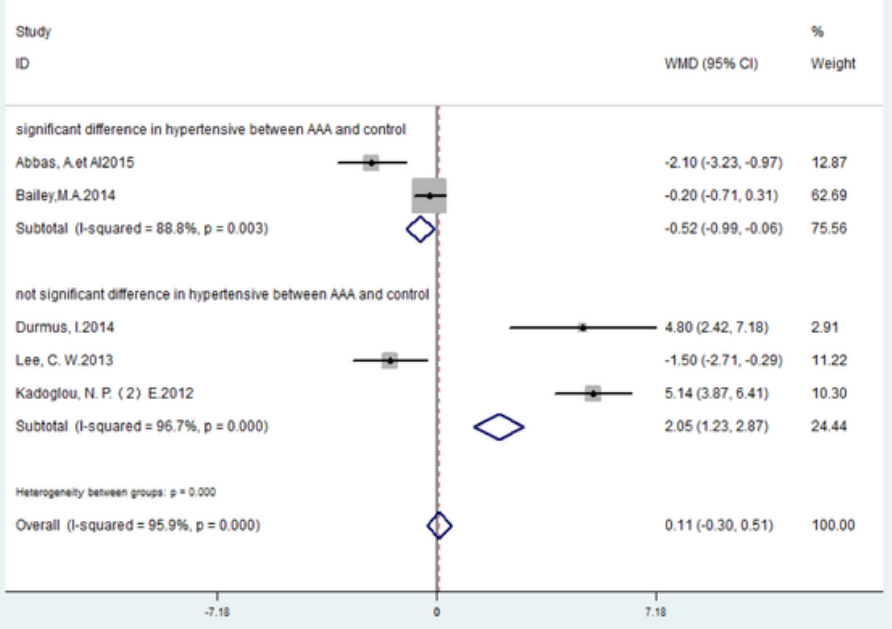

B

study

ID

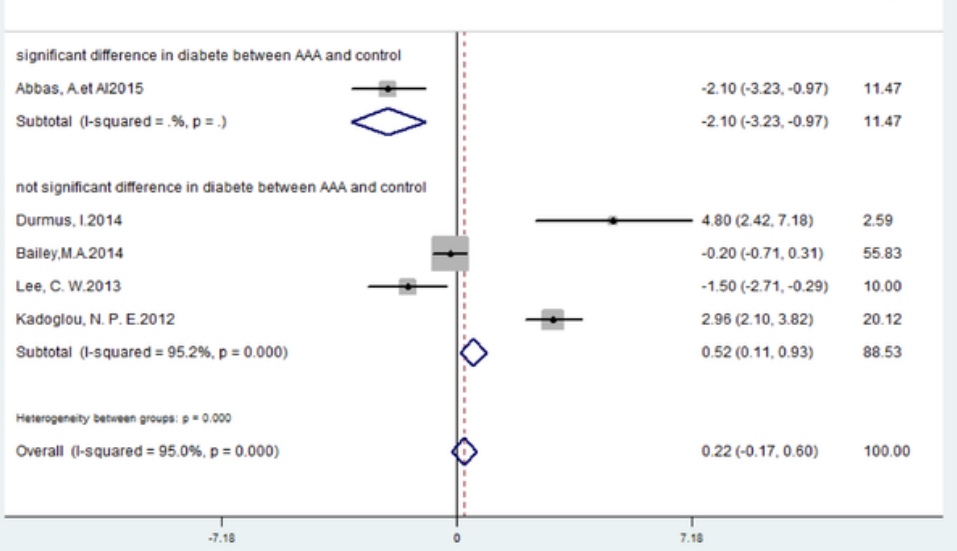

Figure 4

Forest plots of meta-analysis data (A) subgroup analysis according to significant difference or not significant difference in hypertensive between AAA and control group; (B) subgroup analysis according to significant difference or not significant difference in diabete between AAA and control group. 
A

\begin{tabular}{|c|c|c|c|c|}
\hline \multicolumn{3}{|l|}{ Study } & & \multirow{2}{*}{$\%$} \\
\hline ID & & & WMD $(95 \% \mathrm{Cl})$ & \\
\hline \multicolumn{5}{|c|}{ not significant difterence in sex between AAA and control } \\
\hline Abbas, Aet A2015 & $\rightarrow$ & & $-2.10(-3.23,-0.97)$ & 9.04 \\
\hline Durmus, 1.2014 & & $\rightarrow$ & $4.80(2.42,7.18)$ & 2.04 \\
\hline Lee, C. W.2013 & $\rightarrow$ & & $-1.50(-2.71,-0.29)$ & 7.88 \\
\hline Kadoglou, N.P. E.2012 & & + & $2.96(2.10,3.82)$ & 15.86 \\
\hline Kadoglou, N. P. (2) E.2012 & & $\rightarrow$ & $5.14(3.87,6.41)$ & 7.23 \\
\hline LiLL2016 & & & $\mp 12.93(12.02,13.84)$ & 13.95 \\
\hline Subtotal $(1-$ squared $=99.1 \%, p=0.000)$ & & $\Delta$ & $4.35(3.89 .4 .80)$ & 56.00 \\
\hline \multicolumn{5}{|c|}{ significant difference in sex between AAA and control } \\
\hline Bailey,MA2014 & + & & $.0 .20(-0.71,0.31)$ & 44.00 \\
\hline Subtotal (1-squared $=\%, p=$ ) & 8 & & $.0 .20(-0.71,0.31)$ & 44.00 \\
\hline \multicolumn{5}{|l|}{ 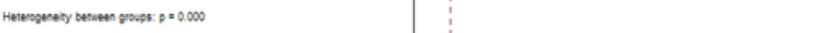 } \\
\hline Overall (1-squared $=99.2 \%, p=0.000$ ) & & $\theta$ & $2.35(2.01,2.69)$ & 100.00 \\
\hline .13 .8 & & & $T_{138}^{T}$ & \\
\hline
\end{tabular}

B Study 10 WMD (95\% Cl) Weight

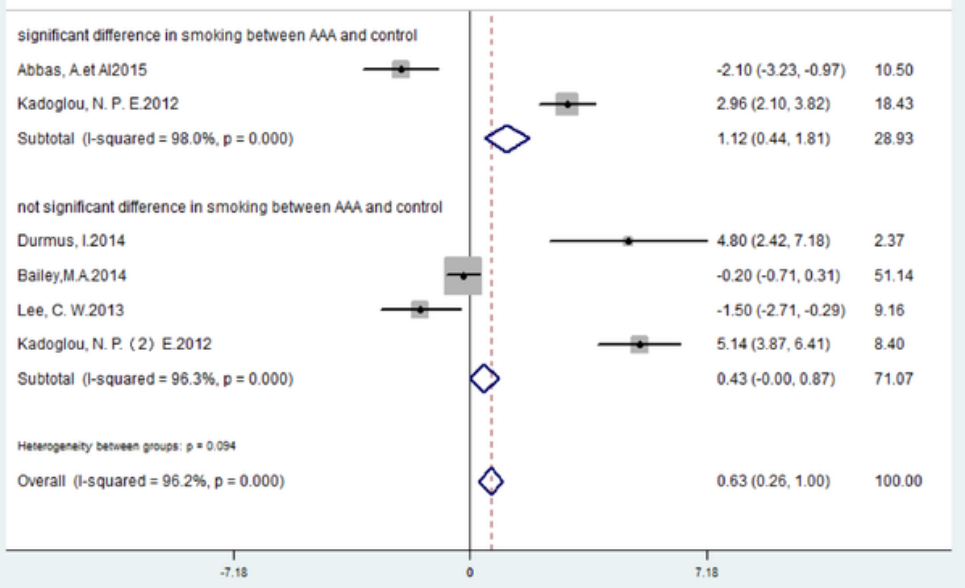

Figure 5

Forest plots of meta-analysis data (A) subgroup analysis according to significant difference or not significant difference in sex between AAA and control group; (B) subgroup analysis according to significant difference or not significant difference in smoking between AAA and control group. 